TRANSACTIONS OF THE

AMERICAN MATHEMATICAL SOCIETY

Volume 362, Number 8, August 2010, Pages 4201-4212

S 0002-9947(10)04976-7

Article electronically published on March 8, 2010

\title{
SOME CONSEQUENCES OF REFLECTION ON THE APPROACHABILITY IDEAL
}

\author{
ASSAF SHARON AND MATTEO VIALE
}

\begin{abstract}
We study the approachability ideal $\mathcal{I}\left[\kappa^{+}\right]$in the context of large cardinals and properties of the regular cardinals below a singular $\kappa$. As a guiding example consider the approachability ideal $\mathcal{I}\left[\aleph_{\omega+1}\right]$ assuming that $\aleph_{\omega}$ is a strong limit. In this case we obtain that club many points in $\aleph_{\omega+1}$ of cofinality $\aleph_{n}$ for some $n>1$ are approachable assuming the joint reflection of countable families of stationary subsets of $\aleph_{n}$. This reflection principle holds under MM for all $n>1$ and for each $n>1$ is equiconsistent with $\aleph_{n}$ being weakly compact in $L$. This characterizes the structure of the approachability ideal $\mathcal{I}\left[\aleph_{\omega+1}\right]$ in models of MM. We also apply our result to show that the Chang conjecture $\left(\kappa^{+}, \kappa\right) \rightarrow\left(\aleph_{2}, \aleph_{1}\right)$ fails in models of MM for all singular cardinals $\kappa$.
\end{abstract}

\section{THE APPROACHABILITY IDEAL}

In the course of development of the pcf-theory of possible cofinalities, Shelah has introduced several interesting stationary sets on the successor of a singular cardinal 1 Among these are the sets of approachable and weakly approachable points in $\kappa^{+}$, where $\kappa$ is a singular cardinal. Given $\mathcal{A}=\left\{a_{\alpha}: \alpha<\kappa^{+}\right\} \subseteq\left[\kappa^{+}\right]^{<\kappa}$, $\delta$ is weakly approachable with respect to $\mathcal{A}$ if there is an $H$ unbounded in $\delta$ of minimal order type such that $\{H \cap \gamma: \gamma<\delta\}$ is covered ${ }^{2}$ by $\left\{a_{\alpha}: \alpha<\delta\right\}$ and $\delta$ is approachable with respect to $\mathcal{A}$ if there is an $H$ unbounded in $\delta$ of minimal order type such that $\{H \cap \gamma: \gamma<\delta\} \subseteq\left\{a_{\alpha}: \alpha<\delta\right\}$.

Definition 1.1. Let $\kappa$ be a singular cardinal. $S$ is (weakly) approachable if there is a sequence $\mathcal{A}=\left\{a_{\alpha}: \alpha<\kappa^{+}\right\} \subseteq\left[\kappa^{+}\right]^{<\kappa}$ and a club $C$ such that $\delta$ is (weakly) approachable with respect to $\alpha$ for all $\delta \in S \cap C$. $\mathcal{I}\left[\kappa^{+}\right]$is the ideal generated by approachable sets; $\mathcal{I}\left[\kappa^{+}, \kappa\right]$ is the ideal generated by weakly approachable sets.

It is clear that $\mathcal{I}\left[\kappa^{+}\right] \subseteq \mathcal{I}\left[\kappa^{+}, \kappa\right]$. For many of the known applications of approachability, it is irrelevant whether we concentrate on the notion of weak approachability or on the apparently stronger notion of approachability. Moreover in the case that $\kappa$ is a strong limit and singular, $\mathcal{I}\left[\kappa^{+}\right]=\mathcal{I}\left[\kappa^{+}, \kappa\right]$ (section 3.4 and proposition 3.23 of [3]). For this reason we feel free to concentrate our attention

Received by the editors April 16, 2008.

2000 Mathematics Subject Classification. Primary 03E04, 03E55; Secondary 03E65.

Key words and phrases. Set theory, singular cardinal combinatorics, large cardinals.

The second author acknowledges support of the Austrian Science Fund FWF project P19375N18 for this research. The second author also thanks Boban Veličković for several useful hints and comments on previous drafts. In particular the results in subsection 2.4 are due to him.

${ }^{1}[3]$ is our main reference source.

${ }^{2}$ I.e.: for every $\gamma<\delta$ there is $\alpha<\delta$ such that $H \cap \gamma \subseteq a_{\alpha}$.

(C)2010 American Mathematical Society 4201 
on the notion of weak approachability which applies to a more general context. It is rather easy to show that $\mathcal{I}\left[\kappa^{+}, \kappa\right]$ is a normal $\kappa^{+}$-closed ideal which extends the non-stationary ideal. A main result of Shelah is that there is a stationary set in $\mathcal{I}\left[\kappa^{+}\right]$for any singular cardinal $\kappa$ (theorem 3.18 in [3]). There are several applications of this ideal to the combinatorics of singular cardinals; we remind the reader of one of them and refer him to section 3 of 3 for a detailed account: the extent of this ideal can be used to size the large cardinal properties of $\kappa . \mathcal{I}\left[\kappa^{+}, \kappa\right]$ is trivial unless the cardinals below $\kappa^{+}$have very strong combinatorial properties (in the range of supercompactness). Thus for example if the square at $\kappa$ holds, then $\mathcal{I}\left[\kappa^{+}\right]=\mathcal{I}\left[\kappa^{+}, \kappa\right]=P\left(\kappa^{+}\right)$(theorem 3.13 of [3]). On the other hand if $\lambda$ is strongly compact and $\kappa>\lambda$ is singular of cofinality $\theta<\lambda$, then there is a stationary subset of $\kappa^{+}$of points of cofinality less than $\lambda$ which is not in $\mathcal{I}\left[\kappa^{+}, \kappa\right]$ (Shelah, theorem 3.20 of [3]). In the same spirit, if MM holds, then there is a stationary set of points of cofinality $\aleph_{1}$ which is not in $\mathcal{I}\left[\aleph_{\omega+1}, \aleph_{\omega}\right]$ (Magidor, unpublished). It is also consistent 3 that for unboundedly many $\alpha<\omega^{2}$ there is a stationary set of points of cofinality $\aleph_{\alpha}$ not in $\mathcal{I}\left[\aleph_{\omega^{2}+1}\right]$. It is an open problem whether it is consistent that there is a stationary set on $\aleph_{\omega+1}$ concentrating on cofinalities larger than $\aleph_{1}$ and not in $\mathcal{I}\left[\aleph_{\omega+1}\right]$ (see for example the introduction of [5] or the end of section 3.5 in (3). We will give a partial answer to this question showing that this is not the case in models of MM. Our results have broader consequences and give serious constraints to the possible scenarios where this problem may have a positive solution. We briefly introduce some relevant concepts in our analysis. $S_{\theta}^{\lambda}$ denotes the subset of $\lambda$ of points of cofinality $\theta$. A stationary subset of $\lambda$ reflects on $\alpha$ if it intersects all the closed and unbounded subsets of $\alpha$.

Definition 1.2. Let $\theta<\lambda$ be regular cardinals.

$R(\lambda, \theta)$ holds for infinite regular cardinals $\theta<\lambda$ if there is $S$, a stationary subset of $\lambda$, such that for all families $\left\{S_{i}: i<\theta\right\}$ of stationary subsets of $S$ there is $\delta<\lambda$ such that $S_{i}$ reflects on $\delta$ for all $i$.

$R^{*}(\lambda)$ holds if there is $S$, a stationary subset of $\lambda$, such that for all families $\left\{S_{i}: i<\lambda\right\}$ of stationary subsets of $S$ there is $\delta<\lambda$ such that $S_{i}$ reflects on $\delta$ for all $i<\delta$.

It is clear that $R^{*}(\lambda)$ implies $R(\lambda, \zeta)$, which implies $R(\lambda, \theta)$ for all $\theta \leq \zeta<\lambda$. Moreover it is not hard to realize $R^{*}(\lambda)$ and $R(\lambda, \theta)$, and we will substantiate this in section 3 We now state one of our main results, which right away gives a clear picture of what we are aiming for. Given regular cardinals $\theta<\lambda, \lambda$ is $\theta$-inaccessible if $\zeta^{\theta}<\lambda$ for all $\zeta<\lambda$.

Theorem 1. Assume:

- $\kappa$ is singular of cofinality $\theta$ and $\nu=\kappa^{+}$,

- $\lambda<\kappa$ is either $\theta$-inaccessible or in $\left[\theta^{+}, \theta^{+\omega}\right)$,

- $R(\lambda, \theta)$ holds.

Then $S_{\lambda}^{\nu} \in \mathcal{I}[\nu, \kappa]$.

Immediate applications of Theorem 1 are the following:

Corollary 2. Assume $\lambda$ is weakly compact, $\kappa>\lambda$ is singular of cofinality $\theta<\lambda$, and $\nu=\kappa^{+}$. Then $S_{\lambda}^{\nu} \in \mathcal{I}[\nu, \kappa]$.

\footnotetext{
${ }^{3}$ See for example [7, where this is achieved in the presence of a very good scale on $\prod_{\alpha<\omega^{2}} \aleph_{\alpha}$.
} 
Proof. $\lambda$ is $\theta$-inaccessible and satisfies $R(\lambda, \theta)$ (see Fact 3.1). Now apply Theorem 1 .

The reflection hypothesis of the main theorem holds in models of strong forcing axioms; for example, we can prove:

Corollary 3. Assume Martin's maximum MM holds. Then club many points in $S_{>\aleph_{1}}^{\aleph_{\omega+1}}$ are approachable.

Proof. MM implies $R\left(\aleph_{n}, \aleph_{1}\right)$ holds as witnessed by $S_{\omega}^{\aleph_{n}}$ for all $n>1$ (see [6]). Now apply Theorem 1 .

We will also be able to obtain by a slight variation of the proof of Theorem 1

Theorem 4. Assume the proper forcing axiom PFA. Then club many points in $S_{>\aleph_{2}}^{\aleph_{\omega+1}}$ are approachable.

Proof. By Theorem 2.15] and Theorem 2.8

Finally in section 4 we will apply these results to the study of the Chang conjecture and prove for example:

Theorem 5. Assume $R\left(\aleph_{2}, \aleph_{1}\right)$. Then $\left(\kappa^{+}, \kappa\right) \rightarrow\left(\aleph_{2}, \aleph_{1}\right)$ fails for all singular $\kappa$.

1.1. Notation and definitions. The paper aims to be accessible and self-contained for any reader with a strong background in combinatorial set theory. While no familiarity with forcing is required, a basic acquaintance with the combinatorics of large cardinals is assumed. When not otherwise explicitly stated, 8] is the standard source for notation and definitions. For a regular cardinal $\theta$, we use $H(\theta)$ to denote the structure $\langle H(\theta), \in,<\rangle$ whose domain is the collection of sets whose transitive closure is of size less than $\theta$ and where $<$ is a predicate for a fixed well ordering of $H(\theta)$. For cardinals $\lambda \leq \kappa$ we let $[\kappa]^{\lambda}$ be the family of subsets of $\kappa$ of size $\lambda$. In a similar fashion we define $[\kappa]^{<\lambda},[\kappa]^{\leq \lambda},[X]^{\lambda}$, where $X$ is an arbitrary set. If $X$ is an uncountable set and $\theta$ is a regular cardinal, $\mathcal{E} \subseteq[X]^{\theta}$ is unbounded if for every $Z \in[X]^{\theta}$, there is $Y \in \mathcal{E}$ containing $Z$. $\mathcal{E}$ is bounded otherwise. For a set of ordinals $X, \bar{X}$ denotes the topological closure of $X$ in the order topology. For regular cardinals $\lambda<\nu, S_{\lambda}^{\nu}$ denotes the subset of $\nu$ of points of cofinality $\lambda$. In a similar fashion, we define $S_{<\lambda}^{\nu}, S_{>\lambda}^{\nu}$, etc. For the ease of the reader we will let $\theta<\lambda<\nu$ range over regular cardinals and $\kappa$ range over singular cardinals in most cases of cofinality $\theta$; moreover, unless otherwise stated, the reader may safely assume that $\nu=\kappa^{+}$. We say that a family $\mathcal{D}$ is covered by a family $\mathcal{E}$ if for every $X \in \mathcal{D}$ there is a $Y \in \mathcal{E}$ such that $X \subseteq Y$.

\section{Covering matrices And the APPROAChability ideal}

Shelah provides a characterization of the ideal $\mathcal{I}\left[\kappa^{+}, \kappa\right]$ which is suitable for our analysis. Let $\kappa$ be singular and let

$$
d:\left[\kappa^{+}\right]^{2} \rightarrow \operatorname{cof}(\kappa) .
$$

- $d$ is normal if $D(i, \beta)=\{\alpha<\beta: d(\alpha, \beta) \leq i\}$ has size less than $\kappa$ for all $i$ and $\beta$,

- $\delta$ is $d$-approachable if there is $H$ unbounded in $\delta$ such that $d\left[[H]^{2}\right]$ is bounded in $\operatorname{cof}(\kappa)$.

The following is an equivalent definition of $\mathcal{I}\left[\kappa^{+}, \kappa\right]$ (theorem 3.28 of [3] $)$. 
Property 2.1. Let $\kappa$ be singular of cofinality $\theta . S \in \mathcal{I}\left[\kappa^{+}, \kappa\right]$ if and only if there are a normal coloring $d$ and a club $C \subseteq \kappa^{+}$such that $\delta$ is $d$-approachable for all $\delta \in S \cap C$.

Proof. We prove only the backward direction, which is the one that we need. So assume $X$ is a subset of $\kappa^{+}$such that for some normal $d$ any $\delta \in X$ is $d$-approachable. Let $D(i, \beta)=\{\alpha<\beta: d(\alpha, \beta) \leq i\}$. We want to define a family $\mathcal{E}=\left\{e_{\alpha}: \alpha<\kappa^{+}\right\}$ such that every point in $X$ is weakly approachable with respect to $\mathcal{E}$. To this aim fix a bijection $\phi: \theta \rightarrow \theta^{2}$ and let $\pi_{0}$ and $\pi_{1}$ be the projection maps of $\theta^{2}$ onto $\theta$. Notice that every ordinal $\delta$ below $\kappa^{+}$can be decomposed uniquely as the sum $\delta=\alpha+i$, where $i<\theta$ and $\alpha$ is divisible by $\theta$. Now for every $\alpha<\kappa^{+}$divisible by $\theta$ and for every $i<\theta$ set $e_{\alpha+i}=D\left(\pi_{0} \circ \phi(i), \alpha+\pi_{1} \circ \phi(i)\right)$. It is not hard to check that if $\delta$ is $d$-approachable, then it is weakly approachable with respect to $\mathcal{E}$.

The coloring $d$ is determined by the matrix $\mathcal{D}(d)=\{D(i, \beta): i<\operatorname{cof}(\kappa), \beta<$ $\left.\kappa^{+}\right\}$, where $D(i, \beta)=\{\alpha<\beta: d(\alpha, \beta) \leq i\}$. It will be convenient for us to treat such matrices instead of the related coloring. Our aim is to show that mild reflection properties of a regular $\lambda<\kappa$ entail that for a suitably chosen normal coloring $d$ all points in $\kappa^{+}$of cofinality $\lambda$ are $d$-approachable. This leads us to introduce and analyze the notion of a covering matrix.

2.1. Covering matrices. The reader is referred to [15] for a detailed account of the results that are mentioned here without proof.

Definition 2.2. For regular cardinals $\theta<\lambda, \mathcal{D}=\{D(i, \beta): i<\theta, \beta<\lambda\}$ is a $\theta$-covering matrix for $\lambda$ if:

(i) $\beta=\bigcup_{i<\theta} D(i, \beta)$ for all $\beta$,

(ii) $D(i, \beta) \subseteq D(j, \beta)$ for all $\beta<\lambda$ and for all $i<j<\theta$,

(iii) for all $\beta<\gamma<\lambda$ and for all $i<\theta$, there is $j<\theta$ such that $D(i, \beta) \subseteq D(j, \gamma)$.

A $\theta$-covering matrix $\mathcal{D}$ is transitive if $\alpha \in D(i, \beta)$ implies $D(i, \alpha) \subseteq D(i, \beta)$.

A $\theta$-covering matrix $\mathcal{D}$ is closed if $\sup X \in D(i, \beta)$ for all $X \in[D(i, \beta)] \leq \theta$.

A $\theta$-covering matrix $\mathcal{D}$ is uniform if for all $\beta<\lambda, D(i, \beta)$ contains a club subset of $\beta$ for eventually all $i<\theta$.

$\beta_{\mathcal{D}} \leq \lambda$ is the least $\beta$ such that for all $i$ and $\gamma, \operatorname{otp}(D(i, \gamma))<\beta$. D is normal if $\beta_{\mathcal{D}}<\lambda$.

Example 2.3. $d:\left[\kappa^{+}\right]^{2} \rightarrow \operatorname{cof}(\kappa)$ is normal if $\mathcal{D}(d)$ is a normal $\operatorname{cof}(\kappa)$-covering matrix on $\kappa^{+}$with $\beta_{\mathcal{D}}=\kappa$.

We will be interested in the matrices produced by the following lemma:

Lemma 2.4. For every singular cardinal $\kappa$, there is a uniform, closed, transitive $\operatorname{cof}(\kappa)$-covering matrix $\mathcal{D}$ on $\kappa^{+}$with $\beta_{\mathcal{D}}=\kappa$.

Proof. Let $\kappa$ be singular of cofinality $\theta$. Fix $\left\{\kappa_{i}: i<\theta\right\}$, an increasing sequence of regular cardinals converging to $\kappa$. Let $\phi_{\alpha}: \kappa \rightarrow \alpha$ be a surjection for all $\alpha<\kappa^{+}$ such that $\phi_{\alpha}\left[\kappa_{i}\right]$ contains a club subset of $\alpha$ whenever $\alpha$ is the limit of cofinality smaller than $\kappa_{i}$. Now set $D_{0}(i, \beta)=\phi_{\beta}\left[\kappa_{i}\right]$ for all $i<\theta$ and $\beta<\kappa^{+}$. Define by recursion over $\xi \leq \theta^{+}$and taking limits and $n<\omega$ :

- $D_{\xi+2 n+1}(i, \beta)=\overline{D_{\xi+2 n}(i, \beta)}$,

- $D_{\xi+2 n+2}(i, \beta)=\bigcup\left\{D_{\xi+2 n+1}(i, \alpha): \alpha \in D_{\xi+2 n+1}(i, \beta)\right\}$,

- $D_{\xi}(i, \beta)=\bigcup\left\{D_{\eta}(i, \beta): \eta<\xi\right\}$. 
Now set $D(i, \beta)=D_{\theta^{+}}(i, \beta)$ and check that $\mathcal{D}=\left\{D(i, \beta): i<\theta, \beta<\kappa^{+}\right\}$is a uniform, closed, transitive $\operatorname{cof}(\kappa)$-covering matrix $\mathcal{D}$ on $\kappa^{+}$with $\beta_{\mathcal{D}}=\kappa$.

Definition 2.5. Let $\mathcal{D}=\{D(j, \beta): j<\theta, \beta<\lambda\}$ be a $\theta$-covering matrix on $\lambda$.

$\operatorname{CP}(\mathcal{D})$ holds if there is $A$, an unbounded subset of $\lambda$, such that $[A]^{\theta}$ is covered by $\mathcal{D}$.

$S(\mathcal{D})$ holds if there is $S$, a stationary subet of $\lambda$, such that for all families $\left\{S_{i}: i<\theta\right\}$ of stationary subsets of $S$ there are $j<\theta$ and $\beta<\lambda$ such that $S_{i} \cap D(j, \beta)$ is non-empty for all $i<\theta$.

We will come back to the relation between approachability and covering matrices at the end of this section. We now aim to investigate the consistency of $S(\mathcal{D})$ and $\mathrm{CP}(\mathcal{D})$ for a large variety of covering matrices $\mathcal{D}$.

\subsection{Consistency of $\mathrm{CP}(\mathcal{D})$ and $S(\mathcal{D})$.}

Fact 2.6. Assume $R(\lambda, \theta)$ holds and that $\mathcal{D}$ is a uniform $\theta$-covering matrix on $\lambda$. Then $S(\mathcal{D})$ holds.

Proof. Let $\mathcal{D}$ be a uniform $\theta$-covering matrix on $\lambda$ and $\left\{S_{i}: i<\theta\right\}$ be a family of stationary subsets of $S$. By $R(\lambda, \theta)$ find $\delta$ such that $S_{i}$ reflects on $\delta$ for all $i<\theta$. Now $\mathcal{D}$ is uniform, so there is a $j<\theta$ such that $D(j, \delta)$ contains a club subset of $\delta$. Thus $S_{i} \cap D(j, \delta)$ is non-empty for all $i<\theta$. Since the family $\left\{S_{i}: i<\theta\right\}$ is arbitrary, $S(\mathcal{D})$ holds as witnessed by $S$.

Corollary 2.7. MM implies $S(\mathcal{D})$ for all uniform $\theta$-covering matrices $\mathcal{D}$ on $\lambda$ whenever $\lambda>\aleph_{1}$ is a regular cardinal and $\aleph_{1} \geq \theta$.

Proof. MM implies that $R\left(\lambda, \aleph_{1}\right)$ holds as witnessed by $S_{\omega}^{\lambda}$ for all regular $\lambda>$ $\aleph_{1}$.

In [15] the following is shown:

Theorem 2.8. PFA implies $\mathrm{CP}(\mathcal{D})$ for every $\omega$-covering matrix $\mathcal{D}$ on a regular $\lambda>\aleph_{2}$.

We now investigate the relation between $\mathrm{CP}(\mathcal{D})$ and $S(\mathcal{D})$ and show that they are equivalent whenever $\mathcal{D}$ is transitive and closed.

\subsection{When are $\operatorname{CP}(\mathcal{D})$ and $S(\mathcal{D})$ equivalent?}

Proposition 2.9. Let $\mathcal{D}$ be a $\theta$-covering matrix on $\lambda$. The following holds:

(i) $\mathrm{CP}(\mathcal{D})$ implies $S(\mathcal{D})$ whenever $\mathcal{D}$ is closed,

(ii) $S(\mathcal{D})$ implies $\mathrm{CP}(\mathcal{D})$ whenever $\mathcal{D}$ is transitive.

Proof. We first show (i). We will actually show that $\operatorname{CP}(\mathcal{D})$ implies $S(\mathcal{D})$ is witnessed by $S_{\theta}^{\lambda}$. So let $\left\{S_{i}: i<\theta\right\}$ be a family of stationary subsets of $S_{\theta}^{\lambda}$. By $\operatorname{CP}(\mathcal{D})$, there is an $X$ unbounded in $\lambda$ such that $[X]^{\theta}$ is covered by $\mathcal{D}$. We claim that $\left[\bar{X} \cap S_{\theta}^{\lambda}\right]^{\theta}$ is covered by $\mathcal{D}$. To see this, let $Z$ be in this latter set and find $Y \subseteq X$ of size $\theta$ such that $Z \subseteq \bar{Y}$. Now find $i$ and $\beta$ such that $Y \subseteq D(i, \beta)$. Since $D(i, \beta)$ is closed under sequences of size at most $\theta, Z \subseteq \bar{Y} \subseteq D(i, \beta)$.

Now pick $M \prec H(\lambda)$ with $\lambda$ large enough such that $|M|=\theta \subseteq M$ and $\theta, X,\left\{S_{i}\right.$ : $i<\theta\} \in M$. Now $S_{i} \cap \bar{X}$ is non-empty for all $i<\theta$. By elementarity, $M$ sees this and so $M \cap S_{i} \cap \bar{X}$ is non-empty for all $i<\theta$. However $M \cap \bar{X} \cap S_{\theta}^{\lambda}$ has size $\theta$ so 
there are $j$ and $\beta$ such that $M \cap \bar{X} \cap S_{\theta}^{\lambda} \subseteq D(j, \beta)$. So $S_{i} \cap D(j, \beta)$ is non-empty for all $i<\theta$. This proves the first implication.

We now show (ii). So assume $S(\mathcal{D})$ holds for a transitive $\theta$-covering matrix $\mathcal{D}$ on $\lambda$. Let $S$ witness $S(\lambda, \theta)$ and $T_{i}$ be the set of $\alpha \in S$ such that

$$
S_{\alpha}^{i}=\{\beta \in S \backslash \alpha: \alpha \in D(i, \beta)\}
$$

is stationary. It is straightforward to see that for some $i<\theta, T_{i}$ is stationary. We aim to show that $\left[T_{i}\right]^{\theta}$ is covered by $\mathcal{D}$ : let $X \in\left[T_{i}\right]^{\theta}$ and consider the family of stationary sets $\left\{S_{\alpha}^{i}: \alpha \in X\right\}$. Since $X$ has size $\theta$, by $S(\mathcal{D})$ there are some $j<\theta$ and $\delta<\lambda$ such that $S_{\alpha}^{i} \cap D(j, \delta)$ is non-empty for all $\alpha \in X$. W.l.og. we can suppose that $j \geq i$. Now for any $\alpha \in X \subseteq T_{i}$, there is $\beta_{\alpha} \in D(j, \delta) \cap S_{\alpha}^{i}$, i.e. $\beta_{\alpha}$ is such that $\alpha \in D\left(i, \beta_{\alpha}\right)$. Since $\mathcal{D}$ is a transitive covering matrix and $j \geq i$,

$$
\alpha \in D\left(i, \beta_{\alpha}\right) \subseteq D\left(j, \beta_{\alpha}\right) \subseteq D(j, \delta) .
$$

This means that $X \subseteq D(j, \delta)$. Since $X$ is arbitrary we can conclude that $\left[T_{i}\right]^{\theta}$ is covered by $\mathcal{D}$.

2.4. A weak form of diagonal reflection. We aim to show that $\operatorname{CP}(\mathcal{D})$ or $S(\mathcal{D})$ strongly limits the kind of behavior a $\theta$-covering matrix $\mathcal{D}$ on $\lambda$ may have. We shall now see that $\operatorname{CP}(\mathcal{D})$ plus suitable assumptions on the proportion between the width $\theta$ and the height $\lambda$ of $\mathcal{D}$ imply that there is an unbounded subset of $\lambda$ such that all its initial segments are covered by $\mathcal{D}$. Once this is achieved, it will be easy to conclude that $R(\lambda, \theta)$ implies that all points of cofinality $\lambda$ below $\kappa^{+}$are weakly approachable whenever $\kappa>\lambda$ is a singular cardinal of cofinality $\theta$. We now prove that a weak form of diagonal reflection of stationary sets on many covering matrices $\mathcal{D}$ follows from $S(\mathcal{D})$ or $\operatorname{CP}(\mathcal{D})$.

Lemma 2.10. Assume $\mathcal{D}$ is a $\theta$-covering matrix on $\lambda, S(\mathcal{D})$ holds as witnessed by $S$ and that either $\lambda$ is $\theta$-inaccessible or $\lambda \in\left(\theta, \theta^{+\omega}\right)$. Then for all families $\left\{S_{\beta}: \beta<\lambda\right\}$ of stationary subsets of $S$ there are $\delta<\lambda$ and $i<\theta$ such that $S_{\alpha} \cap D(i, \delta)$ is non-empty for all $\alpha<\delta$.

Lemma 2.11. Assume $\mathcal{D}$ is a $\theta$-covering matrix on $\lambda, \operatorname{CP}(\mathcal{D})$ holds as witnessed by $T$ and that either $\lambda$ is $\theta$-inaccessible or $\lambda \in\left(\theta, \theta^{+\omega}\right)$. Then there are stationarily many $\delta<\lambda$ such that $T \cap \delta \subseteq D(i, \delta)$ for some $i<\theta$.

We give a detailed proof of the first lemma. The second lemma is proved by a self-evident step-by-step modification of this argument.

Fact 2.12. Let $\theta<\lambda<\nu$ be regular cardinals such that $\lambda^{\theta}<\nu, \mathcal{D}=\{D(j, \beta)$ : $j<\theta, \beta<\nu\}$ a $\theta$-covering matrix on $\nu$ and assume $S(\mathcal{D})$ holds as witnessed by $S$. Let $\left\{S_{i}: i<\lambda\right\}$ be a family of stationary subsets of $S$. Then there are $j<\theta$ and $\beta<\nu$ such that $S_{i} \cap D(j, \beta)$ is non-empty for all $i<\lambda$.

Proof. Let $\left\{S_{i}: i<\lambda\right\}$ be a family of stationary subsets of $S$. We need to find $j<\theta$ and $\beta<\nu$ such that $S_{i} \cap D(j, \beta)$ is non-empty for all $i<\lambda$. For $X \in[\lambda]^{\theta}$, by $S(\mathcal{D})$, we can find $k_{X}<\theta$ and $\beta_{X}<\nu$ such that $S_{i} \cap D\left(k_{X}, \beta_{X}\right)$ is non-empty for all $i \in X$. By our assumptions, $\lambda^{\theta}<\nu$. For this reason, $\beta=\sup _{X \in[\lambda]^{\theta}} \beta_{X}<\nu$. Now by property (ii) of $\mathcal{D}$, we have that for all $X \in[\lambda]^{\theta}, D\left(k_{X}, \beta_{X}\right) \subseteq D\left(j_{X}, \beta\right)$ for some $j_{X}<\theta$. Let $\mathcal{C}_{j}$ be the set of $X$ such that $j_{X}=j$. Now notice that for at least one $j, \mathcal{C}_{j}$ must be unbounded in $[\lambda]^{\theta}$; otherwise $[\lambda]^{\theta}$ would be the union of $\theta$-many bounded subsets, which is not possible since $\lambda$ has cofinality different from 
$\theta$. Then $S_{i} \cap D(j, \beta)$ is non-empty for all $i<\lambda$, since every $i \in \lambda$ is in some $X \in \mathcal{C}_{j}$, as $\mathcal{C}_{j}$ is unbounded. This completes the proof of the fact.

Fact 2.13. Assume $\lambda \in\left(\theta, \theta^{+\omega}\right), \nu>\lambda$ is regular and $S(\mathcal{D})$ holds for some $\theta$ covering matrix $\mathcal{D}$ on $\nu$ and is witnessed by $S$. Let $\left\{S_{i}: i<\lambda\right\}$ be a family of stationary subsets of $S$. Then there are $j<\theta$ and $\beta<\nu$ such that $S_{i} \cap D(j, \beta)$ is non-empty for all $i<\lambda$.

Proof. Proceeding by induction on $n$, assume the claim holds for $\theta^{+n}$ and let $\lambda=$ $\theta^{+(n+1)}$ and $\left\{S_{i}: i<\lambda\right\}$ be a family of stationary subsets of $S$. By the inductive assumption for all $i<\lambda$, there are $k_{i}<\theta$ and $\beta_{i}<\nu$ such that $S_{j} \cap D\left(k_{i}, \beta_{i}\right)$ is nonempty for all $j<i$. Since $\lambda<\nu$ there is $\beta<\nu$ larger than all $\beta_{i}$. Now by property (ii) of $\mathcal{D}$ we have that for all $i<\lambda$ there is $j_{i}<\theta$ such that $D\left(k_{i}, \beta_{i}\right) \subseteq D\left(j_{i}, \beta\right)$. Find $U$, an unbounded subset of $\lambda$, such that for all $i \in U, j_{i}=j$. We can conclude that $S_{l} \cap D(j, \beta)$ is non-empty for all $l<\lambda$, since $S_{l} \cap D\left(k_{i}, \beta_{i}\right)$ is non-empty provided $l<i$ and $i \in U$ and $D\left(k_{i}, \beta_{i}\right) \subseteq D(j, \beta)$.

We are now ready to prove Lemma 2.10

Proof. Assume not and let $\left\{S_{\beta}: \beta<\nu\right\}$ contradict the lemma. For each $\delta$ of cofinality larger than $\theta$, let $\gamma_{\delta}<\delta$ be the least such that for all $i<\theta$ there is $\gamma_{i}^{\delta}<\gamma_{\delta}$ such that $S_{\gamma_{i}^{\delta}} \cap D(i, \delta)$ is empty. Find $A$, a stationary subset of $\nu$, such that $\gamma_{\delta}=\gamma$ for all $\delta \in A$. By our assumption on $\nu$ and Facts 2.12 and 2.13, we know that there are $i<\theta$ and $\delta_{0}<\nu$ such that $S_{\alpha} \cap D\left(i, \delta_{0}\right)$ is non-empty for all $\alpha<\gamma$. Pick $\delta \in A \backslash \delta_{0}$ and $j<\theta$ such that $D\left(i, \delta_{0}\right) \subseteq D(j, \delta)$. Then we get that $S_{\gamma_{j}^{\delta}} \cap D(j, \delta)$ is non-empty since $S_{\gamma_{j}^{\delta}} \cap D\left(i, \delta_{0}\right)$ is non-empty and $D\left(i, \delta_{0}\right) \subseteq D(j, \delta)$. This contradicts the very definition of $\gamma_{j}^{\delta}$.

In particular we have shown the following:

Fact 2.14. Assume $\lambda$ is either $\theta$-inaccessible or $\lambda \in\left(\theta, \theta^{+\omega}\right)$ and $S(\mathcal{D})$ holds for a transitive $\theta$-covering matrix $\mathcal{D}$ on $\lambda$. Then there is $A$, an unbounded subset of $\lambda$, such that $[A]^{<\lambda}$ is covered by $\mathcal{D}$.

2.5. Main result. We are now in a position to state our main result:

Theorem 2.15. Assume $\kappa$ is singular of cofinality $\theta$ and a regular $\lambda<\kappa$ is either $\theta$-inaccessible or in $\left(\theta, \theta^{+\omega}\right)$ and such that $S(\mathcal{D})$ (or equivalently $\operatorname{CP}(\mathcal{D})$ ) holds for all uniform, closed and transitive $\theta$-covering matrices $\mathcal{D}$ on $\lambda$. Then club many points in $\kappa^{+}$of cofinality $\lambda$ are approachable.

Proof. Fix $d:\left[\kappa^{+}\right]^{2} \rightarrow \theta$ such that $D(d)=\left\{D(i, \beta): i<\theta, \beta<\kappa^{+}\right\}$is a normal, uniform, closed and transitive $\theta$-covering matrix on $\kappa^{+}$, where $D(i, \beta)=\{\alpha<\beta$ : $d(\alpha, \beta) \leq i\}$. Such a $d$ exists by Lemma 2.4. By Property 2.1] it is enough to show that all points of cofinality $\lambda$ are $d$-approachable. Let $\beta$ be such that $\operatorname{cof}(\beta)=\lambda$. Find $A=\left\{\delta_{\xi}: \xi<\lambda\right\}$, a closed and unbounded subset of $\beta$ of minimal order-type. Let $\pi$ be the transitive collapse of $A$ on $\lambda$ and $\mathcal{E}=\{E(i, \xi): i<\theta, \xi<\lambda\}$ be the matrix whose entries are the sets $\pi\left[D\left(i, \delta_{\xi}\right) \cap A\right]$. Then $\mathcal{E}$ is a uniform, closed and transitive $\theta$-covering matrix on $\lambda$. By $S(\mathcal{E})$ and Fact 2.14, there is $B$, an unbounded subset of $\lambda$, such that $[B]^{<\lambda}$ is covered by $\mathcal{E}$. Thus $B \cap \eta \subseteq E\left(i_{\eta}, \xi_{\eta}\right)$ for some $i_{\eta}<\theta$ and $\xi_{\eta} \in B \backslash \eta$ for all $\eta \in B$. Refine $\left\{\xi_{\eta}: \eta \in B\right\}$ to an unbounded subset $C$ such that $\xi_{\eta}<\gamma$ for all $\xi_{\eta}<\xi_{\gamma} \in C$. Thus $\xi_{\eta} \in B \cap \gamma \subseteq E\left(i_{\gamma}, \xi_{\gamma}\right)$ for all $\xi_{\eta}<\xi_{\gamma} \in C$. Let $D$ be an unbounded subset of $C$ such that for some fixed $j$, 
$i_{\eta}=j$ for all $\xi_{\eta} \in D$. Now if $\xi_{\eta}<\xi_{\gamma} \in D$ we have that $\xi_{\eta} \in B \cap \gamma \subseteq E\left(j, \xi_{\gamma}\right)$, i.e. $d\left(\pi^{-} 1\left(\xi_{\eta}\right), \pi^{-1}\left(\xi_{\gamma}\right)\right) \leq j$, i.e. $\pi^{-1}[D]$ witnesses that $\beta$ is $d$-approachable.

\section{JOINT REFLECTION OF STATIONARY SETS}

We briefly analyze the consistency strength of the hypothesis of Theorem 1

Fact 3.1. $R^{*}(\lambda)$ holds if $\lambda$ is weakly compact.

Proof. Recall the following characterization of weak compactness: $\lambda$ is weakly compact if for every transitive model $M$ of ZFC minus the powerset axiom such that $M$ has size $\lambda$ and $H(\lambda) \subseteq M$, there is an elementary embedding of $M$ into a transitive structure $N$ with critical point $\lambda$. Now let $\left\{S_{i}: i<\lambda\right\}$ be any family of stationary subsets of $\lambda$. To prove $R^{*}(\lambda)$ we must find a $\delta<\lambda$ such that $S_{\alpha}$ reflects on $\delta$ for all $\alpha<\delta$. Let $M$ be a structure as above such that $\left\{S_{i}: i<\lambda\right\} \in M$. Let $j: M \rightarrow N$ be elementary with $N$ transitive and a critical point of $j=\lambda$. Then $j\left(\left\{S_{\alpha}: \alpha<\lambda\right\}\right)=\left\{T_{\alpha}: \alpha<j(\lambda)\right\}$ and $j\left(S_{\alpha}\right) \cap \lambda=S_{\alpha}$ for all $\alpha<\lambda$. Thus $N$ models that there is $\delta<j(\lambda)$ (namely $\delta=\lambda$ ) such that for all $\alpha<\delta, T_{\alpha}$ reflects on $\delta$. By elementarity of $j$ there is $\delta<\lambda$ such that $S_{\alpha}$ reflects on $\delta$ for all $\alpha<\delta$ and we are done.

Larson (unpublished) has proved that MM implies $R^{*}\left(\aleph_{2}\right)$ while it is apparent already in the paper of Foreman, Magidor and Shelah [6] that MM implies $R\left(\lambda, \aleph_{1}\right)$ for all regular $\lambda>\aleph_{1}$. On the other hand, Magidor [11] has shown that $R^{*}\left(\aleph_{n}\right)$ is equiconsistent with $\aleph_{n}$ being weakly compact in $L$. Notice however that models of $R\left(\aleph_{n}, \aleph_{0}\right)$ and $R\left(\aleph_{n+1}, \aleph_{0}\right)$ subsume already very large cardinal assumptions since it can be seen that $R\left(\aleph_{n}, \aleph_{0}\right)$ implies failure o $\square\left(\aleph_{n}\right)$ and Schimmerling has shown that failure of $\square\left(\aleph_{n}\right)$ for two consecutive cardinals implies projective deteminacy 12. Another scenario suggested by Foreman to obtain $R^{*}(\lambda)$ is the following:

Lemma 3.2. Assume that $\mathcal{I}$ is a $\lambda$-complete, fine ideal which concentrates on $[\kappa]<\lambda$ for some $\kappa \geq \lambda$ and such that $P_{\mathcal{I}}=P\left([\kappa]^{<\lambda}\right) / \mathcal{I}$ is a proper forcing. Then $R^{*}(\lambda)$ holds.

Proof. First of all $\mathcal{I}$ is precipitous since $P_{\mathcal{I}}$ is proper (4, Proposition 4.10). Let $G$ be a generic filter for $P_{\mathcal{I}}$. Then the ultrapower $M=V^{\left([\kappa]^{<\lambda}\right)} \cap V / G$ defined in $V[G]$ is well-founded. Let $j: V \rightarrow M$ be the associated generic elementary embedding. Since $\mathcal{I}$ is $\lambda$-complete and fine, we have that the critical point of $j$ is $\lambda$. Now let $\left\{S_{\alpha}: \alpha<\lambda\right\} \in V$ be a family of stationary subsets of $S_{\aleph_{0}}^{\lambda}$. It is clear that $M$ models that $j\left(\left\{S_{\alpha}: \alpha<\lambda\right\}\right)=\left\{T_{\alpha}: \alpha<j(\lambda)\right\}$ is a family of stationary subsets of $S_{\aleph_{0}}^{j(\lambda)}$. Now $T_{\alpha}=j\left(S_{\alpha}\right)$ and $j\left(S_{\alpha}\right) \cap \lambda=S_{\alpha}$ for all $\alpha<\lambda$. Since $P$ is proper, $S_{\alpha}$ is a stationary subset of $\lambda$ in $V[G]$, so it is certainly a stationary subset of $\lambda$ in $M$. Then $M$ models that $j\left(S_{\alpha}\right)$ reflects on $\lambda$ for all $\alpha<\lambda$. Now the argument to show that $S^{*}(\lambda)$ holds in $V$ is as in Fact 3.1 .

Notice that we've hidden a large cardinal assumption in the requirement that $P$ is proper. The hypotheses of the lemma are satisfied by the non-stationary ideal on $\aleph_{2}$ in the generic extension by a Levy collapse of a measurable $\lambda$ to $\aleph_{2}$. In this case the quotient algebra is even countably complete. [4] is a survey on generic

\footnotetext{
${ }^{4}$ For example, Todorčević produces from $\square(\lambda)$ a transitive and closed $\omega$-covering matrix $\mathcal{D}$ on $\lambda$ such that $\operatorname{CP}(\mathcal{D})$ cannot hold (see section 7 and lemma 7.1 .10 of 13 or, for a more detailed proof, section 2.2.1 of [14]).
} 
large cardinals. We now turn to an application of Theorem 1 and Theorem 2.15 to Chang conjectures.

\section{4. $R\left(\aleph_{2}, \aleph_{1}\right)$ DENIES $\left(\kappa^{+}, \kappa\right) \rightarrow\left(\aleph_{2}, \aleph_{1}\right)$ FOR ALL SINGULAR CARDINALS $\kappa$}

Recall that the Chang conjecture $(\lambda, \kappa) \rightarrow(\theta, \nu)$ holds for $\lambda>\kappa \geq \theta>\nu$ if for every structure $\langle Y, \lambda, \kappa, \ldots\rangle$ with predicates for $\lambda$ and $\kappa$ there is $X \prec Y$ such that $|X \cap \lambda|=\theta$ and $|X \cap \kappa|=\nu$. We will also be interested in the principles of the form $(\kappa, \lambda) \rightarrow(\theta,<\theta)$ which are likewise defined. It is known that $\left(\aleph_{2}, \aleph_{1}\right) \rightarrow\left(\aleph_{1}, \aleph_{0}\right)$ as well as many other Chang conjectures is consistent relative to appropriate assumptions of large cardinals. For example it is possible to see that $\left(j\left(\kappa^{+\theta}\right), j\left(\kappa^{+\gamma}\right)\right) \rightarrow\left(\kappa^{+\theta}, \kappa^{+\gamma}\right)$ whenever $\kappa$ is the critical point of a 2-huge embedding and $\gamma<\theta<\kappa$. Developing on this, Levinski, Magidor and Shelah in [10] showed that $\left(\aleph_{\omega+1}, \aleph_{\omega}\right) \rightarrow\left(\aleph_{1}, \aleph_{0}\right)$ is consistent relative to the existence of a 2-huge cardinal. However, all the known examples of a consistent $\left(\kappa^{+}, \kappa\right) \rightarrow\left(\theta^{+}, \theta\right)$ where $\kappa$ is singular and $\theta$ regular are such that $\theta=\operatorname{cof}(\kappa)$. Thus a folklore problem in this field is the following:

Problem 6. Is it consistent that $\left(\kappa^{+}, \kappa\right) \rightarrow\left(\theta^{+}, \theta\right)$ for some regular $\theta$ and singular $\kappa$ of cofinality smaller than $\theta$ ?

First of all it is a simple fact that such Chang conjectures affect cardinal arithmetic:

Fact 4.1. Assume $\left(\kappa^{+}, \kappa\right) \rightarrow\left(\theta^{+}, \theta\right)$ for some singular $\kappa$. Then $\theta^{+} \leq \theta^{\operatorname{cof}(\kappa)}$.

Proof. Notice that $\kappa^{\operatorname{cof}(\kappa)}>\kappa$. Now assume $\left(\kappa^{+}, \kappa\right) \rightarrow\left(\theta^{+}, \theta\right)$. Fix $\lambda>\kappa^{+}$regular and large enough and let $H(\lambda)$ denote the family of sets whose transitive closure has size less than $\theta$. Fix $M \prec\left\langle H(\lambda), \kappa^{+}, \kappa, \ldots.\right\rangle$ with $\left|M \cap \kappa^{+}\right|=\theta^{+}$and $|M \cap \kappa|=\theta$. Pick a family $\left\{X_{\alpha}: \alpha<\kappa^{+}\right\} \in M$ of distinct elements of $[\kappa]^{\operatorname{cof}(\kappa)}$. By elementarity of $M, X_{\alpha} \cap M \neq X_{\beta} \cap M$ for all $\alpha, \beta \in M \cap \kappa^{+}$. Thus we have a family of $\theta^{+}$ distinct elements of $[M \cap \kappa]^{M \cap \operatorname{cof}(\kappa)}$. Now $|M \cap \kappa|=\theta$ and $|M \cap \operatorname{cof}(\kappa)| \leq \operatorname{cof}(\kappa)$. Thus $\theta^{+} \leq\left|[M \cap \kappa]^{M \cap \operatorname{cof}(\kappa)}\right| \leq \theta^{\operatorname{cof}(\kappa)}$.

Cummings in 2] has shown that these Chang conjectures can be studied by means of pcf-theory and has obtained several other restrictions on the combinatorics of the singular cardinals $\kappa$ which may satisfy an instance of the above problem. For example he has shown that these Chang conjectures subsume the existence of very strong large cardinals, i.e. out of the scope of analysis of the current inner model theory: it can be argued by the analysis brought up in [2] that $\left(\aleph_{\omega+1}, \aleph_{\omega}\right) \rightarrow$ $\left(\aleph_{n}, \aleph_{n-1}\right)$; then $\square_{\aleph_{\omega}}$ fails and $\mathrm{SCH}$ holds at $\aleph_{\omega}$. Moreover a result by Shelah shows that $n$ cannot be greater than 3 . We can decrease 3 down to 1 and greatly simplify their argument avoiding any mention of scales in the case that $R\left(\aleph_{2}, \aleph_{1}\right)$ holds:

Theorem 4.2. Assume $R\left(\aleph_{2}, \aleph_{1}\right)$ holds. Then $\left(\kappa^{+}, \kappa\right) \rightarrow\left(\aleph_{2}, \aleph_{1}\right)$ fails for all singular cardinals $\kappa$.

\footnotetext{
${ }^{5}$ Cummings' analysis relies on the notion of good (or in Kojamn's notation 9 flat) points for a scale on $\prod_{n} \aleph_{n}$ and his main observation (Lemma 3.1 of [2]) is that if $\left(\aleph_{\omega+1}, \aleph_{\omega}\right) \rightarrow\left(\aleph_{n}, \aleph_{n-1}\right)$ holds, then there are stationarily many non-good points of cofinality $\aleph_{n}$. On the other hand, Shelah has shown that club many points of cofinality $\aleph_{n}$ are good (or flat) for a scale on $\prod_{n} \aleph_{n}$ if either $\aleph_{n}>2^{\aleph_{0}}$ (Exercise 2.9-2, Lemma 2.12 and Theorem 2.13 of [1]) or $n>3$ (Theorem 2.13 and Lemmas 2.12 and 2.19 of [1]).
} 
Proof. Fix $M \prec H(\Gamma)$, a structure containing all relevant information and such that $|M \cap \kappa|=\aleph_{1}$ and $\left|M \cap \kappa^{+}\right|=\aleph_{2}$. First of all:

Claim 4.3. otp $\left(M \cap \kappa^{+}\right)=\aleph_{2}$.

Suppose otherwise and let $\gamma \in M$ be such that $\operatorname{otp}(M \cap \gamma)=\aleph_{2}$. Then $\gamma \in$ $M \cap\left(\kappa, \kappa^{+}\right)$. We claim that $M$ models that $\gamma$ is a regular cardinal, which gives the desired contradiction, since by elementarity $\gamma$ would be in the universe a regular cardinal in $\left(\kappa, \kappa^{+}\right)$, which is impossible. So suppose $M$ models that $\gamma$ is not a cardinal. Then in $M$ there is a bijection $\phi$ of $\gamma$ onto $\kappa$. If we take the transitive collapse $\pi_{M}$ of $M, \pi_{M}(\phi)$ is now a bijection of $\pi_{M}(\gamma)=\aleph_{2}$ onto $\pi_{M}(\kappa)$, which is an ordinal of size less than $\aleph_{2}$. This contradiction proves the claim.

Fix in $M$ a transitive and uniform $\operatorname{cof}(\kappa)$-covering matrix $\mathcal{D}$ on $\kappa^{+}$, where $\beta_{\mathcal{D}}=$ $\kappa$. Let $X=M \cap \kappa^{+}$and $\delta_{M}=\operatorname{otp}(M \cap \kappa)$. This is one key observation:

Fact 4.4. If $C \in M$ is a closed set of ordinals and $\pi_{M}$ is the transitive collapse of $M$, then $\pi_{M}(C)$ is a closed set of ordinals.

Let $\alpha_{M}=M \cap \operatorname{cof}(\kappa)$. Now otp $(M \cap \operatorname{cof}(\kappa))<\operatorname{otp}(M \cap \kappa)<\aleph_{2}$. So $\operatorname{cof}\left(\alpha_{M}\right)=$ $\aleph_{i} \leq \aleph_{1}$. Let $\phi: \aleph_{i} \rightarrow M \cap \alpha_{M}$ be strictly increasing and cofinal and set

$$
\mathcal{E}_{0}=\left\{\pi_{M}(D(\phi(j), \alpha)): j<\aleph_{i}, \alpha \in M \cap \kappa^{+}\right\} .
$$

Claim 4.5. $\mathcal{E}_{0}$ is a transitive and uniform $\omega$-covering matrix on $\aleph_{2}$ with $\beta_{\mathcal{D}} \leq \delta_{M}$.

Proof. Transitivity of $\mathcal{E}$ is immediate to check. $\mathcal{E}$ is uniform due to the above fact and the elementarity of $M$ : for every $\beta \in M \cap \kappa^{+}, \operatorname{cof}(\beta) \in M$ by elementarity of $M$. Since $\mathcal{D}$ is uniform, $D(\xi, \beta)$ contains a club subset $C_{\beta}$ of $\beta$ for eventually all $\xi<\operatorname{cof}(\kappa)$. By elementarity of $M$ the least such $\xi<\operatorname{cof}(\kappa)$ is in $M$ and there is such a club $C_{\beta} \in M$. Now $\pi_{M}\left(C_{\beta}\right)$ is a club subset of $\pi_{M}(D(\phi(j), \beta))$ for all $j$ such that $\phi(j)>\xi$. The bound on the order type of the entries on $\mathcal{E}$ follows by the fact that $M \models \operatorname{otp}(D(\xi, \beta))<\kappa$ for all $\xi<\alpha_{M}$ and $\beta \in M \cap \kappa^{+}$, so $\pi_{M}[M] \models \operatorname{otp}\left(\pi_{M}(D(\phi(i), \beta))\right)<\delta_{M}$ for all $i<\aleph_{i}$.

Now $\mathcal{E}_{0}=\left\{E(j, \beta): j<\aleph_{i}, \beta<\aleph_{2}\right\}$ is a transitive, uniform, $\aleph_{i}$-covering matrix on $\aleph_{2}$ with $\beta_{\mathcal{E}} \leq \delta_{M}<\aleph_{2}$. By $R\left(\aleph_{2}, \aleph_{1}\right), S\left(\mathcal{E}_{0}\right)$ holds, so there is $A$ unbounded in $\aleph_{2}$ such that $[A]^{\aleph_{i}}$ is covered by $\mathcal{E}$. By Lemma 2.11, $[A]^{\aleph_{1}}$ is covered by $\mathcal{D}$. So find $\gamma$ such that $A \cap \gamma$ has order type greater than $\delta_{M}$. Now there are $j<\aleph_{i}$ and $\beta<\aleph_{2}$ such that $A \cap \gamma \subseteq E(j, \beta)$. Then:

$$
\delta_{M}<\operatorname{otp}(A \cap \gamma) \leq \operatorname{otp}(E(j, \beta))<\delta_{M} .
$$

This is the desired contradiction, which proves the theorem.

4.1. MM denies many other instances of $\left(\kappa^{+}, \kappa\right) \rightarrow\left(\aleph_{2}, \aleph_{1}\right)$. We briefly recall an argument presumably due to Silver which shows that under PFA, $\left(\kappa^{+}, \kappa\right) \rightarrow$ $\left(\aleph_{2}, \aleph_{1}\right)$ fails for all regular $\kappa$ such that $\kappa^{<\kappa}=\kappa$. Under PFA this cardinal arithmetic assumption holds for $\kappa=\aleph_{2}$.

Definition 4.6. $(T,<)$ is a tree if it is a well-founded partial order with a least element called the root of $T$ and such that for any $a \in T, \operatorname{pr}(a)=\{b \in T: b<a\}$ is a well-ordered subset of $T$ under $<. a, b \in T$ are compatible if $a \leq b$ or $b \leq a$. $A \subseteq T$ is an antichain if any two elements of $A$ are incompatible.

- $\operatorname{ht}(a)=\operatorname{otp}(\operatorname{pr}(a),<)$,

- $\operatorname{Lev}_{\alpha}(T)=\{a: \operatorname{ht}(a)=\alpha\}$ is the $\alpha$-th level of $T$, 
- $\mathrm{ht}(T)=\sup \{\mathrm{ht}(a): a \in T\}$,

- $B \subseteq T$ is an $\alpha$-branch if $(B,<)$ is a well order and $\sup \{\operatorname{ht}(a): a \in B\}=\alpha$,

- $(T,<)$ is special if $\mathrm{ht}(T)=\kappa^{+}$and $T$ is the union of $\kappa$-many antichains,

- $T$ is a $\lambda$-tree if all its levels have size less than $\lambda$,

- $T$ is Aronszjain if it is a $\lambda$-tree and has no $\lambda$-branches. Note that a special $\kappa^{+}$-tree is Aronszjain.

- $\lambda$ has the tree property if there is no Aronszjain tree of height $\lambda$.

Theorem 4.7 (Specker). If $\kappa^{<\kappa}=\kappa$, there is a special $\kappa^{+}$-tree.

Theorem 4.8 (Baumgartner). Assume PFA for posets of size continuum. Then $\aleph_{2}$ has the tree property.

Now assume $\kappa^{<\kappa}=\kappa$ and $\left(\kappa^{+}, \kappa\right) \rightarrow\left(\aleph_{2}, \aleph_{1}\right)$. Let $M \prec H\left(\kappa^{++}\right)$be a structure such that $\operatorname{otp}\left(M \cap \kappa^{+}\right)=\aleph_{2}$. Let $T \in M$ be a special $\kappa^{+}$-tree as witnessed by the specializing function $f$. Let $\pi_{M}$ be the transitive collapse of $M$. It is possible to check that $\pi_{M}(T)$ is a special $\aleph_{2}$-tree as witnessed by the specializing function $\pi_{M}(f)$. This contradicts the tree property on $\aleph_{2}$, which follows from PFA.

\section{Some open QUestions And SOME COMments}

The original question by Magidor and Foreman [5] remains open:

Problem 7. Is it consistent that $S_{\aleph_{2}}^{\aleph_{\omega+1}} \notin \mathcal{I}\left[\aleph_{\omega+1}\right]$ ?

Foreman and Cummings have independently shown that it is possible to force right away in ZFC by a cardinal preserving forcing a transitive, uniform $\omega$-covering matrix on $\aleph_{2}$ such that $S(\mathcal{D})$ fails. Veličković noticed that it is possible to obtain further counterexamples to $S(\mathcal{D})$ using Todorčević's techniques of minimal walks over a $\square\left(\aleph_{2}\right)$-sequence 6 So the reflection hypotheses on $\aleph_{2}$ are needed to obtain that $S(\mathcal{D})$ holds for every uniform and transitive $\omega$-covering matrix $\mathcal{D}$ on $\aleph_{2}$. On the other hand no strategy to force $S_{\aleph_{2}}^{\aleph_{\omega+1}} \notin \mathcal{I}\left[\aleph_{\omega+1}\right]$ seems currently available.

A negative answer to the above problem would also entail a negative answer to question 6, i.e.:

Problem 8. Is it consistent that $\left(\aleph_{\omega+1}, \aleph_{\omega}\right) \rightarrow\left(\aleph_{2}, \aleph_{1}\right)$ ?

It seems more fruitful to attack this problem directly by means of Shelah's analysis of the existence of exact upper bounds for families of ordinal functions in $O r d^{\omega}$ (see 2 and section 2 of [1). For example, using these techniques, we can already prove that $\left(\aleph_{\omega+1}, \aleph_{\omega}\right) \rightarrow\left(\aleph_{n}, \aleph_{n-1}\right)$ fails if $n>3$.

A comment on our Main Theorem 1 is in order: the theorem entails that in a model of $\mathrm{MM}, S_{\lambda}^{\kappa^{+}} \in \mathcal{I}\left[\kappa^{+}, \kappa\right]$ for all $\kappa$ of countable cofinality and for all regular $\lambda<\kappa$ which are $\omega$-inaccessible, i.e., which are not the successor of a cardinal of countable cofinality. We expect this to be close to the best possible result for models of MM. For example consider the following scenario: $\kappa$ is a supercompact cardinal and $\left(\lambda^{+\omega+1}, \lambda^{+\omega}\right) \rightarrow\left(\nu^{+\omega+1}, \nu^{+\omega}\right)$ for some $\nu>\kappa$ holds as witnessed by structures $M$ such that $M^{\kappa} \subseteq M$. This occurs if there is a 2-huge cardinal larger than $\kappa$. Now force MM collapsing $\kappa$ to $\aleph_{2}$. In the resulting generic extension, MM holds and the chain condition of the forcing is small enough to preserve the

\footnotetext{
${ }^{6}$ Todorčević's book 13 gives a complete account of the method of minimal walks and of its applications.
} 
truth of $\left(\lambda^{+\omega+1}, \lambda^{+\omega}\right) \rightarrow\left(\nu^{+\omega+1}, \nu^{+\omega}\right)$. This Chang conjecture already implies that $S_{\nu+\omega+1}^{\lambda^{+\omega+1}} \notin \mathcal{I}\left[\lambda^{+\omega+1}\right]$.

Finally the results of the last subsection suggest the following problem:

Problem 9. Is it at all consistent with MM that $\left(\kappa^{+}, \kappa\right) \rightarrow\left(\aleph_{2}, \aleph_{1}\right)$ holds for some $\kappa ?$

If $\mathrm{GCH}$ holds above $\aleph_{2}, \kappa^{<\kappa}=\kappa$ for all regular $\kappa \geq \aleph_{2}$. This assumption is certainly consistent with $\mathrm{MM}$ and is incompatible with $\left(\kappa^{+}, \kappa\right) \rightarrow\left(\aleph_{2}, \aleph_{1}\right)$ in the presence of PFA.

\section{REFERENCES}

1. U. Abraham and M. Magidor, Cardinal arithmetic, Handbook of Set Theory (M. Foreman, A. Kanamori, and M. Magidor, eds.), North Holland, to appear.

2. J. Cummings, Collapsing successors of singulars, Proceedings of the American Mathematical Society 125(9) (1997), 2703-2709. MR.1416080 (97j:03091)

3. T. Eisworth, Successors of singular cardinals, Handbook of Set Theory (M. Foreman, A. Kanamori, and M. Magidor, eds.), North Holland, to appear.

4. M. Foreman, Ideals and Generic Elementary Embeddings, Handbook of Set Theory (M. Foreman, A. Kanamori, and M. Magidor, eds.), North Holland, to appear.

5. M. Foreman and M. Magidor, A very weak square principle, Journal of Symbolic Logic 62(1) (1997), 175-196. MR.1450520 (98i:03062)

6. M. Foreman, M. Magidor, and S. Shelah, Martin's Maximum, saturated ideals and nonregular ultrafilters, Annals of Mathematics (2) 127(1) (1988), 1-47. MR.924672 (89f:03043)

7. M. Gitik and A. Sharon, On SCH and the approachability property, Proceedings of the American Mathematical Society 136 (2008), 311-320. MR.2350418 (2008m:03102)

8. T. Jech, Set theory, The Third Millennium Edition, Revised and Expanded, Springer, 2002. MR.1940513 (2004g:03071)

9. M. Kojman, Exact upper bounds and their uses in set theory, Annals of Pure and Applied Logic 92 (1998), 267-282. MR1640912 (2000b:03163)

10. J.-P. Levinski, M. Magidor, and S. Shelah, Chang's conjecture for $\aleph_{\omega}$, Israel Journal of Mathematics 69(2) (1990), 161-172. MR1045371 (91g:03071)

11. M. Magidor, Reflecting stationary sets, Journal of Symbolic Logic 62(4) (1982), 755-771. MR683153 (84f:03046)

12. E. Schimmerling, Coherent sequences and threads, Advances in Mathematics 216 (2007), 89-117. MR2353251 (2009b:03141)

13. S. Todorčević, Walks on Ordinals and Their Characteristics, Birkhäuser, 2007. MR 2355670

14. M. Viale, Application of the proper forcing axiom to cardinal arithmetic, Ph.D. thesis, Université Paris 7-Denis Diderot, 2006.

15. _ A family of covering properties, Mathematical Research Letters 15(2) (2008), 221238. MR2385636 (2009b:03130)

TARAd 11, Apt. 10, 52503 Ramat Gan, Israel

Dipartimento di Matematica, Università di Torino, via Carlo Alberto 10, 10123 TORINO, ITALY

E-mail address: matteo.viale@unito.it 